\title{
BIOCHEMICAL EVALUATION OF ANTIDIABETIC PROPERTIES OF SWERTIAMARIN, A SECOIRIDOID GLYCOSIDE OF ENICOSTEMMA LITTORALE LEAVES, STUDIED IN HIGH-FAT DIET-FED LOW-DOSE STREPTOZOTOCIN-INDUCED TYPE 2 DIABETIC RATS
}

\author{
SELVAM R, MURUGANANTHAM K, SUBRAMANIAN S* \\ Department of Biochemistry, University of Madras, Guindy Campus, Chennai - 600 025, Tamil Nadu, India. \\ Email: subbus2020@yahoo.co.in
}

Received: 27 June 2018, Revised and Accepted: 21 August 2018

ABSTRACT

Objective: Swertiamarin, a secoiridoid glycoside present in the leaves of Enicostemma littorale, is reported to be responsible for its pharmacological and beneficial properties. The present study was aimed to biochemically evaluate the antidiabetic properties of Swertiamarin in high fat diet fed - low dose streptozotocin (STZ)-treated diabetic rats.

Methods: High-fat diet-fed low-dose STZ was used to induce experimental type 2 diabetes in rats. Diabetic rats were orally treated with swertiamarin (50 mg/kg b.w./rat/day) for 30 days. The physiological criterions such as food and fluid intake were recorded. Oral glucose tolerance test was performed. The levels of fasting blood glucose, plasma insulin, glycosylated hemoglobin A1c (HbA1c), hemoglobin, and homeostasis model assessment of insulin resistance (HOMA-IR) values were estimated. The activities of key enzymes involved in carbohydrate and glycogen metabolism in the liver and kidney tissues were assayed. The glycogen content in liver tissue was estimated.

Result: Oral administration of swertiamarin to diabetic rats established a significant decline in the levels of fasting blood glucose, HbA1c as well as HOMA-IR values and an increase in plasma insulin and hemoglobin levels. The altered activities of key enzymes of carbohydrate and glycogen metabolism in liver and kidney tissues of diabetic rats were restored to near normalcy by swertiamarin treatment.

Conclusion: Swertiamarin treatment maintains normoglycemia in diabetic rats by modulating the activities of key carbohydrate and glycogen metabolizing enzymes in the hepatic and renal tissues.

Keywords: Swertiamarin, High-fat diet, Streptozotocin, Antidiabetic property.

(C) 2018 The Authors. Published by Innovare Academic Sciences Pvt Ltd. This is an open access article under the CC BY license (http://creativecommons. org/licenses/by/4. 0/) DOI: http://dx.doi.org/10.22159/ajpcr.2018.v11i10.28152

\section{INTRODUCTION}

Last few decades witnessed a rapid increase in the incidence of diabetes mellitus due to increased socioeconomic developments in many parts of the world. Type 2 diabetes mellitus (T2DM) accounts for more than $90 \%$ of the diabetic population globally and is chiefly manifested by insulin resistance which in turn arises due to obesity. The influence of obesity and other contributing factors in inducing T2DM has been reported in many studies around the world. Its incidence is increasing alarmingly owing to genetic and environmental factors which ultimately results in decreased insulin synthesis, impaired regulation of glucose metabolism, and pancreatic $\beta$-cell dysfunction. Obesity and insulin resistance have been found to play an integral role in the initiation and progression of T2DM. Insufficient insulin secretion results in reduced glucose transport into the vital organs such as liver, muscle, and adipose tissues [1]. In the initial stages of diabetes mellitus, the chronic hyperglycemia induces excessive secretion of insulin leading to hyperinsulinemia and subsequently to hypoinsulinemia [2]. The chronic hyperglycemia is reported to play a pivotal role in all forms of secondary complications of diabetes. The onset of T2DM is progressive, and it involves glucotoxicity and lipotoxicity commonly known as glucolipotoxicity [3].

Glucose is a principal source of energy for all cells and organs. However, chronic elevation of its levels results in diabetes and its secondary complications. Blood glucose levels should be maintained within the physiological range. The liver plays an important role in buffering the blood glucose level by contributing either hepatic glucose utilization or hepatic glucose production (HGP) depending on the prevailing plasma glucose level to maintain the critical threshold value of $\sim 6 \mathrm{mM}$. The hepatocytes are equipped with an array of regulatory mechanisms to control the process of glycogenesis, glycogenolysis, glycolysis, and gluconeogenesis $[4,5]$. There are several options to maintain normoglycemia in diabetic individuals. However, most of the currently available antidiabetic medications elicit undesirable side effects and diminution in response after prolonged use [6]. Hence, search for newer drugs has become a continuous process and the medicinal plants serve as a major source of drug discovery.

Among the various medicinal plants which have been traditionally used for the treatment of diabetes, Enicostemma littorale Blume which belongs to the family of Gentianaceae plays an imperative role. The leaf extract is used in reducing obesity and improving the liver functions [7]. Swertiamarin, a secoiridoid glycoside, originally isolated from the leaves of $E$. littorale and was reported to be responsible for the medicinal properties of $E$. littorale. It forms as an important constituent of many herbal preparations in Japan $[8,9]$.

Li et al. reported the absorption, distribution, and elimination pattern of swertiamarin [10]. Several reports are available in the literature regarding the pharmacological properties of swertiamarin. However, most of the reports are pertaining to in vitro experiments only. Further, the mechanism of action of swertiamarin has not been explored at a systemic level $[11,12]$. More recently, we have reported the phytochemical analysis and antioxidant potentials of E. littorale leaf extract [13]. In the absence of systemic studies in the literature pertaining to the antidiabetic properties of swertiamarin, in the present study, an attempt has been made to evaluate the antidiabetic property of swertiamarin in HFD-fed low-dose STZ-induced type 2 diabetes in rats. 


\section{MATERIALS AND METHODS}

\section{Chemicals}

Streptozotocin (STZ) and swertiamarin were procured from Sigma Aldrich, stored at $2^{\circ} \mathrm{C}$ to $4^{\circ} \mathrm{C}$, and protected from light. All other chemicals used in the present study were purchased from standard commercial suppliers and were of analytical grade quality.

\section{Acute toxicity and dosage fixation studies}

Acute toxicity studies were performed as per the OECD guidelines (423) for testing of chemicals in normal rats. Graded doses of swertiamarin dissolved in $0.2 \%$ dimethyl sulfoxide were administered orally. The rats were observed for 4 weeks following oral administration. The changes in food consumption, fluid intake, psychomotor activities, changes in body weight gain, and changes in skin, fur, eyes, salivation, diarrhea, and lethargy were consistently monitored. Macroscopic examination of vital organs was also performed. Similarly, the dosage fixation studies were carried out by administering graded doses of swertiamarin $(5,10,20,25$, and $50 \mathrm{mg} / \mathrm{kg}$ b.w./rat/day) for 30 days to determine the dose-dependent hypoglycemic effect in HFD-fed lowdose STZ-induced diabetic rats by monitoring the fasting blood glucose levels periodically.

\section{Experimental animals}

Male Wistar Albino rats weighing about $160-180 \mathrm{~g}$, procured from Tamilnadu Veterinary and Animal Sciences University, Chennai, India, were housed in clean, sterile, polypropylene cages under standard vivarium conditions $\left(12 \mathrm{~h}\right.$ light/dark cycle) and temperature $\left(25 \pm 2^{\circ} \mathrm{C}\right)$ with ad libitum access to water and standard rat chow (Hindustan Lever Ltd., Bengaluru, India) with a composition of $5 \%$ fat, $21 \%$ protein, $55 \%$ nitrogen-free extract, and $4 \%$ fiber $(\mathrm{w} / \mathrm{w})$ with adequate minerals and vitamins for the animals. The animals were acclimatized to the laboratory conditions for 2 weeks before the inception of experiments. The studies were conducted strictly according to the rules and norms stipulated by the Institutional Animal Ethics Committee Guidelines.

\section{High-fat diet (HFD)-fed low-dose STZ-induced diabetes}

The rats were divided into two dietary regimens by feeding either normal or HFD for the initial period of 2 weeks [14]. The ingredients and chemical composition of the HFD was followed and is mentioned in Table 1.

After 2 weeks of dietary manipulation to develop insulin resistance, the groups of rats fed with HFD were intraperitoneally injected with a freshly prepared low dose of STZ (35 mg/kg b.w) dissolved in $0.1 \mathrm{M}$ ice-cold citrate buffer, $\mathrm{pH}$ 4.5. 3 days after injection with STZ, the experimental rats were screened for fasting blood glucose level. Experimental rats which show the fasting blood glucose above $250 \mathrm{mg} / \mathrm{dl}$ were chosen for further studies. The rats were allowed to continue to feed on the respective diets until the end of the experiments.

\section{EXPERIMENTAL PROTOCOL}

The rats were divided into four groups each group comprising six rats. Group 1: Control rats

Group 2: Diabetic rats (HFD low-dose STZ; 35 mg/kg b.w.)

Group 3: Diabetic rats treated with swertiamarin $(50 \mathrm{mg} / \mathrm{kg}$ b.w.)

Group 4: Diabetic rats treated with metformin ( $50 \mathrm{mg} / \mathrm{kg}$ b.w.).

During the experimental period, body weight, blood glucose, food and water consumption, and physical examinations were determined at regular intervals. The dosage was adjusted every week according to any change in body weight to maintain similar dose per kg body weight of rat over the entire period of study for each group. At the end of the treatment period, the rats were fasted overnight, anesthetized (ketamine, $80 \mathrm{mg} / \mathrm{kg}$ b.w., i.p.), and sacrificed by cervical decapitation. The blood was collected with or without anticoagulants for plasma or serum separation, respectively. The liver, kidney, and pancreatic tissues were dissected out and washed in ice-cold saline and used for further experimental studies.
Oral glucose tolerance test (OGTT)

On the day before sacrifice, an OGTT was performed in all the groups. Blood samples were obtained from the lateral tail vein of rats deprived of food overnight. Successive blood samples were taken at $0,30,60,90$, and $120 \mathrm{~min}$ following the oral administration of $2 \mathrm{~g} / \mathrm{kg} \mathrm{b.w}$. of glucose solution.

Homeostasis model assessment of insulin resistance (HOMA-IR) Insulin resistance was evaluated by HOMA-IR by the method of Matthews et al. [15], as follows:

$$
\text { HOMA IR }=\frac{\text { Flood Glucose }(\mathrm{mg} / \mathrm{dl})}{405}
$$

\section{Biochemical parameters}

The levels of blood glucose were measured by glucose oxidase method [16]. Plasma insulin was assayed using ELISA kit for rats (Linco Research, Inc., USA). The levels of hemoglobin and glycosylated hemoglobin A1c (HbA1c) were estimated $[17,18]$. Urine sugar was detected using urine strips.

\section{Assay of key enzymes of carbohydrate metabolism}

A portion of the liver and kidney tissues was dissected, washed immediately with ice-cold saline, and homogenized in $0.1 \mathrm{M}$ Tris$\mathrm{HCl}$ buffer $(\mathrm{pH}$ 7.4) for the assay of key enzymes of carbohydrate metabolism. The debris was removed by centrifugation at 10,000 rpm, and the supernatant was used for the assay of hexokinase, pyruvate kinase (PK), lactate dehydrogenase (LDH), glucose-6-phosphatase, fructose-1,6-bisphosphatase, glucose-6-phosphate dehydrogenase, glycogen synthase, and glycogen phosphorylase activity [19-26]. Glycogen content was estimated in wet liver tissue [27].

\section{Statistical analysis}

The results were expressed as mean \pm SEM of six rats in each group, and the statistical significance was evaluated by one-way Analysis of Variance (ANOVA) using the SPSS (version 16) program followed by least significant difference (LSD). Values were considered statistically significant when $\mathrm{p}<0.05$.

\section{RESULTS}

The levels of food and fluid intake in control and experimental groups of rats are shown in Figs. 1 and 2, respectively. HFD-STZ-induced diabetic rats showed a significant $(\mathrm{p}<0.05)$ increase in food and fluid intake when compared with the control group of rats. On oral administration of swertiamarin to the diabetic group of rats, the levels were found to be similar to that of the control group of rats, and the efficacy was comparable with metformin.

The levels of blood glucose in certain specific durations after the oral administration of glucose $(2 \mathrm{~g} / \mathrm{kg} \mathrm{b.w}$.) in control and the experimental group of rats are depicted in Fig. 3. In control group of rats, the blood glucose level reached the maximum peak at $60 \mathrm{~min}$ after an oral glucose load, and then, it was gradually reverted back to near normal levels

Table 1: Composition of the high fat diet

\begin{tabular}{ll}
\hline Ingredients & Diet (g/kg) \\
\hline Powdered NPD* & 365 \\
Lard & 310 \\
Casein & 250 \\
Cholesterol & 10 \\
Vitamin and mineral mix & 60 \\
DL-methionine & 03 \\
Yeast powder & 01 \\
Sodium chloride & 01 \\
\hline
\end{tabular}

*The composition of normal pellet diet (NPD): $4.1 \%$ fat, $22.2 \%$ protein, and $12.1 \%$ carbohydrates, as a percentage of total kilocal 
after 120 min indicating the maintenance of normoglycemia, whereas in the case of STZ-induced diabetic group of rats, the peak increase in blood glucose concentration was observed after $60 \mathrm{~min}$ and remained high over the next $60 \mathrm{~min}$. Oral administration of swertiamarin, as well as metformin to HFD-STZ, induced diabetic rats showed a significant decrease in blood glucose concentration at 60 and 120 min when compared with the diabetic group of rats.

Fig. 4 represents the homeostatic assessment of insulin resistance (HOMA-IR) in the control and experimental groups of rats. A significant elevation in HOMA-IR values was observed in the diabetic group of rats. On oral administration of swertiamarin, HOMA-IR values were significantly decreased, and the effect was found to be comparable with metformin.

The effect of oral administration of swertiamarin on the levels of fasting blood glucose, plasma insulin, and HbA1c in HFD-fed low-dose STZinduced experimental type 2 diabetic rats is presented in Table 2 . In the diabetic group of rats, the levels of fasting blood glucose and HbA1c were significantly increased with a concomitant decrease in the levels of plasma insulin and hemoglobin. Treatment with swertiamarin significantly decreased the altered levels of fasting blood glucose and HbA1c in diabetic groups of rats. The plasma insulin and hemoglobin levels were improved to the physiological range on oral administration with swertiamarin for 30 days.

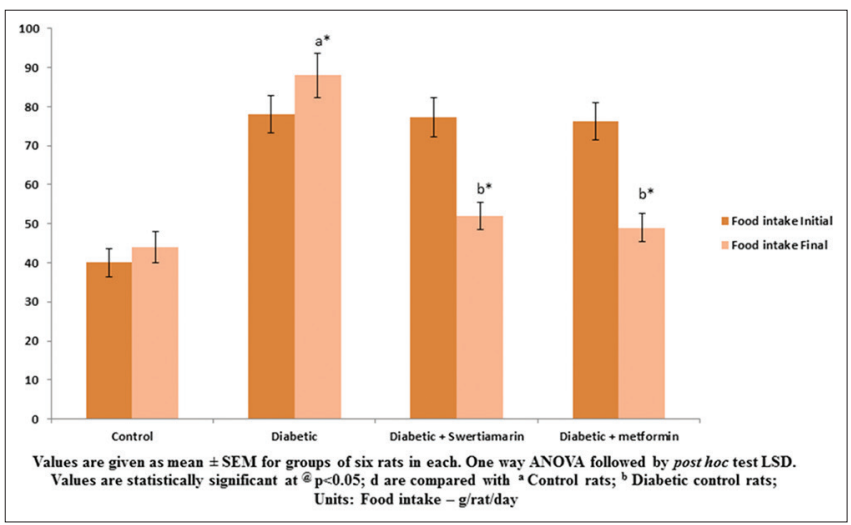

Fig. 1: Effect of swertiamarin on food intake of experimental groups of rats. Values are given as mean \pm SEM for groups of six rates in each. One-way ANOVA followed by post-hoc test LSD. Values are statistically significant at ${ }^{\circledR} \mathbf{p}<0.05$; ${ }^{\mathrm{d}}$ are compared with ${ }^{a}$ control rats, ${ }^{b}$ diabetic control rats, units: Food intake - g/rat/day

SEM: Standard error mean, LSD: Least significant difference

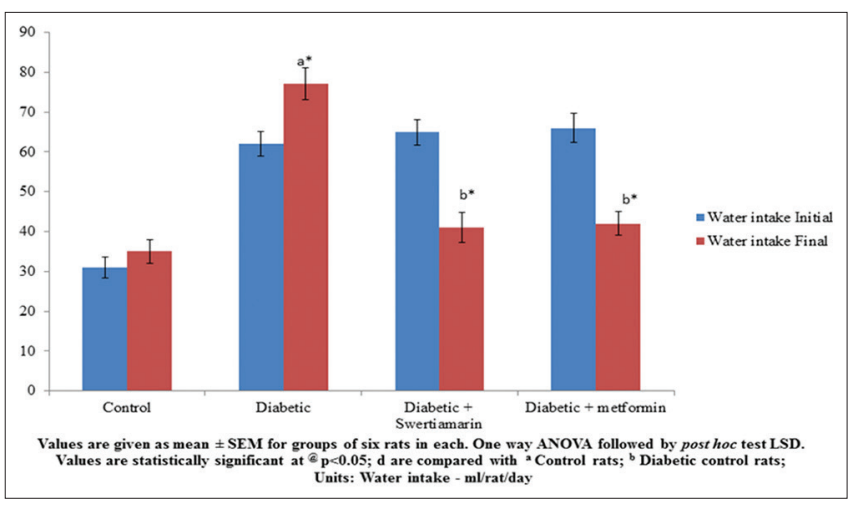

Fig. 2: Effect of Swertiamarin on water intake of experimental groups of rats. Values are given as mean \pm SEM for groups of six rates in each. One-way ANOVA followed by post-hoc test LSD. Values are statistically significant at ${ }^{\circledR} \mathrm{p}<0.05$, dare compared with ${ }^{a}$ control rats, bdiabetic control rats, units: Water intake - $\mathrm{ml} / \mathrm{rat}$ / day. SEM: Standard error mean, LSD: Least significant difference
The activities of regulatory enzymes such as hexokinase, PK, and LDH in liver and kidney tissues of control and experimental groups of rats are presented in Tables 3 and 4. It is evident from the data that the activities of both hexokinase and PK were significantly decreased in the hepatic tissue of diabetic rats. Swertiamarin treatment modulates the activity of the enzymes in hepatic tissues of HFD-fed STZ-induced diabetic rats. The activity of LDH in diabetic rats was significantly elevated when compared to control rats. Altered activity of LDH in diabetic rats is reverted to near normal values on oral administration with swertiamarin as well as metformin.

Tables 5 and 6 depict the activities of glucose-6-phosphatase, fructose-1, 6-bisphosphatase, and glucose-6-phosphate dehydrogenase in liver and kidney tissues of control and experimental groups of rats. Experimental type 2 diabetic rats showed significantly increased activities of glucose-6-phosphatase and fructose-1, 6-bisphosphatase. The activity of glucose-6-phosphate dehydrogenase was significantly decreased in diabetic rats. These altered activities were bringing back to normal levels by oral administration of Swertiamarin. Metformintreated diabetic rats also exhibit similar results.

Table 7 shows the level of liver glycogen and the activities of glycogen synthase and glycogen phosphorylase in control and experimental

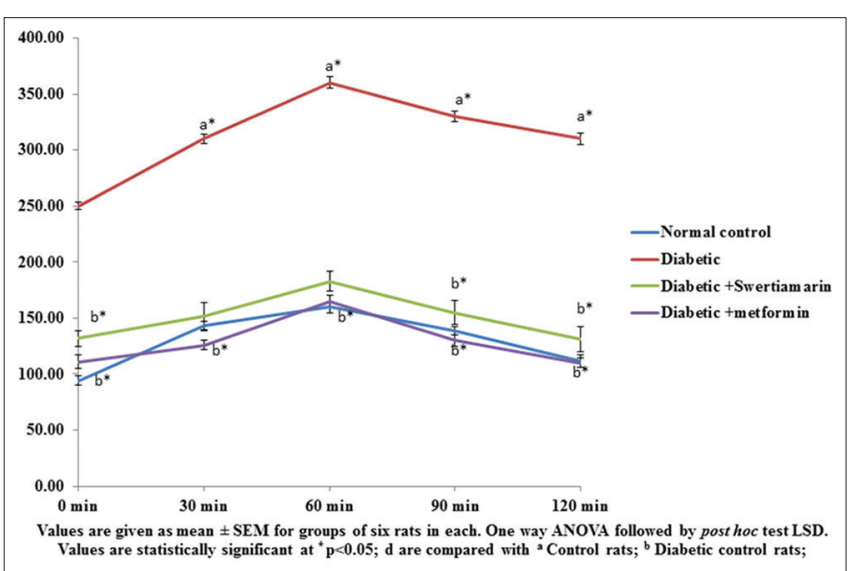

Fig. 3: Oral glucose tolerance test of control and experimental groups of rats. Values are given as mean \pm SEM for groups of six rates in each. One-way ANOVA followed by post-hoc test LSD. Values are statistically significant at ${ }^{\circledR} \mathbf{p}<0.05$, dare compared with ${ }^{a}$ control rats, bdiabetic control rats. SEM: Standard error mean, LSD: Least significant difference

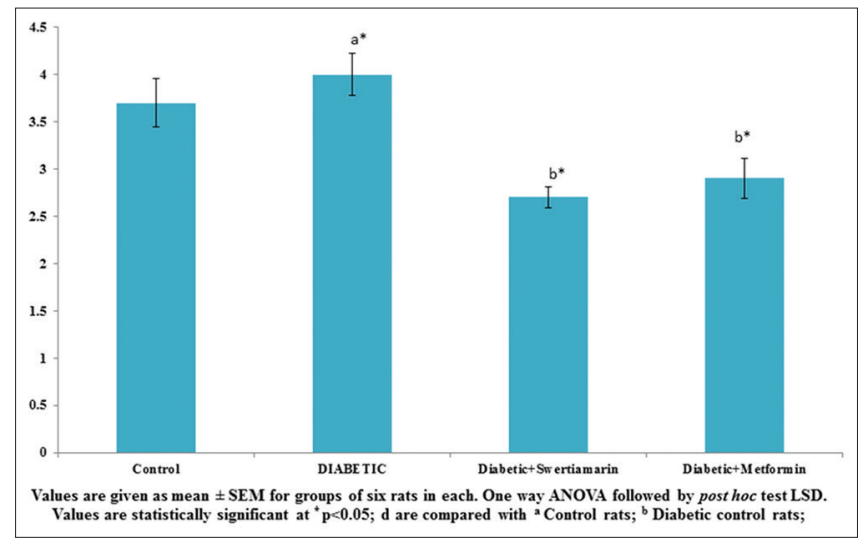

Fig. 4: Homeostasis model assessment of insulin resistance of control and experimental groups of rats. Values are given as mean \pm SEM for groups of six rates in each. One-way ANOVA followed by post-hoc test LSD. Values are statistically significant at ${ }^{\circledR} \mathbf{p}<0.05$, dare compared with acontrol rats, ${ }^{b}$ diabetic control rats.

SEM: Standard error mean, LSD: Least significant difference 
Table 2: The levels of blood glucose, hemoglobin, HbA1c, plasma insulin, and urine sugar in control and experimental groups of rats

\begin{tabular}{llllll}
\hline Groups & Blood glucose & Hemoglobin & HbA1c & Insulin & Urine sugar \\
\hline Control & $92.50 \pm 3.17$ & $12.40 \pm 0.27$ & $6.11 \pm 0.21$ & $16.30 \pm 0.45$ & $\mathrm{Nil}$ \\
Diabetic & $288.33 \pm 9.33^{\mathrm{a} *}$ & $10.40 \pm 0.43^{\mathrm{a} *}$ & $13.60 \pm 0.32^{\mathrm{a} *}$ & $5.52 \pm 0.26^{\mathrm{a} *}$ & +++ \\
Diabetic+swertiamarin & $138.00 \pm 5.25^{\mathrm{b} *}$ & $13.11 \pm 0.28^{* *}$ & $7.80 \pm 0.24^{\mathrm{b} *}$ & $8.20 \pm 0.43^{\mathrm{b} *}$ & $\mathrm{Nil}$ \\
Diabetic+metformin & $128.50 \pm 4.00^{\mathrm{b} *}$ & $12.43 \pm 0.49^{\mathrm{b} *}$ & $7.21 \pm 0.22^{\mathrm{b*}}$ & $9.40 \pm 0.48^{\mathrm{b*}}$ & $\mathrm{Nil}$ \\
\hline
\end{tabular}

Units: $\mathrm{mg} / \mathrm{dl}$ for blood glucose, $\mathrm{g} / \mathrm{dl}$ for hemoglobin, \% hemoglobin for HbA1c, $\mu \mathrm{U} / \mathrm{ml}$ for plasma insulin, +++indicates more than $2 \%$ sugar. Results are expressed as mean \pm SEM $(n=6)$. One-way ANOVA followed by post-hoc test LSD. Values are statistically significant at ${ }^{*} \mathrm{p}<0.05$. The results were compared with ${ }^{\mathrm{a}} \mathrm{control}$ rats, ${ }^{\mathrm{b}} \mathrm{diabetic}$ rats. SEM: Standard error mean, LSD: Least significant difference, HbA1c: Glycosylated hemoglobin A1c

Table 3: Effect of swertiamarin on the activities of hexokinase and PK in liver tissues of control and experimental groups of rats

\begin{tabular}{llll}
\hline Groups & Hexokinase & PK \\
\hline Control & $279.62 \pm 8.33$ & $230.00 \pm 9.02$ \\
Diabetic & $150.23 \pm 7.03^{\mathrm{a} *}$ & $123.80 \pm 4.42^{\mathrm{a} *}$ & $209.00 \pm 3.03$ \\
Diabetic+swertiamarin & $216.10 \pm 9.68^{\mathrm{b} *}$ & $185.40 \pm 6.32^{\mathrm{b} *}$ & $532.25 \pm 33.24^{\mathrm{a} *}$ \\
Diabetic+metformin & $232.82 \pm 9.52^{\mathrm{b} *}$ & $176.10 \pm 5.20^{\mathrm{b} *}$ & $300.50 \pm 5.10^{\mathrm{b} *}$ \\
\hline
\end{tabular}

Units are expressed as: $\mu$ moles of glucose-6-phosphate formed/h/mg of protein for hexokinase, $\mathrm{mU} / \mathrm{mg}$ of protein for PK. Values are given as mean \pm SEM ( $\mathrm{n}=6$ ) for

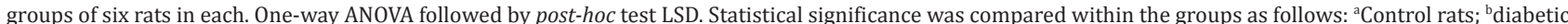
control rats; Values are statistically significant at *p<0.05. SEM: Standard error mean, LSD: Least significant difference, PK: Pyruvate kinase, LDH: Lactate dehydrogenase

Table 4: Effect of swertiamarin on the activities of hexokinase and PK in kidney tissues of control and experimental groups of rats

\begin{tabular}{llll}
\hline Groups & Hexokinase & PK & LDH \\
\hline Control & $152.50 \pm 5.00$ & $175.00 \pm 7.80$ & $510.15 \pm 15.00$ \\
Diabetic & $83.27 \pm 5.00^{\mathrm{a}}$ & $100.00 \pm 5.50^{\mathrm{a} *}$ & $729.10 \pm 24.10^{\mathrm{a} *}$ \\
Diabetic+swertiamarin & $114.40 \pm 6.60^{\mathrm{b} *}$ & $135.00 \pm 8.40^{\mathrm{b} *}$ & $550.20 \pm 20.21^{\mathrm{b*}}$ \\
Diabetic+metformin & $123.00 \pm 5.60^{\mathrm{b*}}$ & $142.50 \pm 6.70^{\mathrm{b*}}$ & $542.00 \pm 21.32^{\mathrm{b*}}$ \\
\hline
\end{tabular}

Units are expressed as: $\mu$ moles of glucose-6-phosphate formed/h/mg of protein for hexokinase, $\mathrm{mU} / \mathrm{mg}$ of protein for PK, $\mu$ moles of pyruvate formed/h/mg of protein for lactate dehydrogenase. Values are given as mean \pm SEM for groups of six rats in each. One-way ANOVA followed by post-hoc test LSD. Statistical significance was compared within the groups as follows: ${ }^{a}$ Control rats; ${ }^{b}$ diabetic control rats. Values are statistically significant at ${ }^{*} \mathrm{p}<0.05$. SEM: Standard error mean, LSD: Least significant difference, PK: Pyruvate kinase, LDH: Lactate dehydrogenase

Table 5: Activities of glucose-6-phosphatase, fructose-1, 6-bisphosphatase, and glucose-6-phosphate dehydrogenase in liver tissues of control and experimental groups of rats

\begin{tabular}{llll}
\hline Groups & Glucose-6-phosphatase & Fructose-1,6-bisphosphatase & Glucose-6-phosphate dehydrogenase \\
\hline Control & $873.30 \pm 29.20$ & $354.00 \pm 16.05$ & $483.00 \pm 29.33$ \\
Diabetic & $1580.40 \pm 68.80^{\mathrm{a} *}$ & $833.20 \pm 34.08^{\mathrm{a} *}$ & $265.10 \pm 16.20^{\mathrm{a} *}$ \\
Diabetic+swertiamarin & $1050.29 \pm 43.00^{\mathrm{b} *}$ & $468.72 \pm 25.70^{\mathrm{b} *}$ & $392.42 \pm 20.20^{\mathrm{b} *}$ \\
Diabetic+metformin & $1100.10 \pm 50.8^{\mathrm{b} *}$ & $460.50 \pm 20.50^{0^{*}}$ & $350.50 \pm 22.80^{\mathrm{b} *}$ \\
\hline
\end{tabular}

Units are expressed as: $\mu$ moles of Pi liberated/h/mg of protein for glucose-6-phosphatase and fructose-1, 6-bisphosphatase and $\mu$ moles of NADPH/min/mg of protein for glucose-6-phosphate dehydrogenase. Values are given as mean \pm SEM for groups of six rats in each. One-way ANOVA followed by post-hoc test LSD. Statistical significance was compared within the groups as follows: ${ }^{a}$ control rats; ${ }^{b}$ diabetic control rats. Values are statistically significant at * $<0.05$. SEM: Standard error mean, LSD: Least significant difference

groups of rats. Diabetic rats showed a considerable decrease in the liver glycogen content and glycogen synthase activity and a concomitant increase in glycogen phosphorylase activity. Oral administration of swertiamarin to the diabetic group of rats restored the level of liver glycogen and the activities of glycogen synthase, as well as glycogen phosphorylase, were brought back to physiological levels.

\section{DISCUSSION}

Phytotherapy continues to show a better complimentary potential in the treatment of various free radical-mediated diseases such as diabetes and cancer. The bioactive principles present in various parts of plants serve as potential antioxidants in preventing and/or protecting against several diseases in humans [28]. Phytochemicals derived from medicinal plants are widely used as raw materials for drug development. However, only a few traditionally important medicinal plants have been subjected to pharmacological scrutiny. One such phytochemical which lacks scientific validation for its traditional beneficial and pharmacological properties is Swertiamarin, a secoiridoid glycoside originally isolated from the leaves of $E$. littorale.
Feeding with HFD is routinely used to develop insulin resistance in experimental animals [29-32]. Administration of low dose of STZ ( $35 \mathrm{mg} / \mathrm{kg}$ b.w.) to HFD-fed rats causes partial destruction of pancreatic $\beta$-cells by necrosis, resulting in insulin deficiency [33]. Srinivasan et al. reported that the combination of HFD-fed and low-dose STZ-treated rats serve an alternative animal model simulating the natural disease progression and metabolic characteristics of type 2 diabetes, and the model is widely used to screen the therapeutic potential of compounds exhibiting insulin-sensitizing and secretory effects.

Type 2 diabetes induced by high-fat diet is associated with obesity, hyperglycemia, and hyperinsulinemia which induces $\beta$-cell dysfunction and excessive generation of free radicals [34]. $\beta$-cell dysfunction and insulin resistance are intrinsically complex with their interrelation for inducing the pathogenesis of type 2 diabetes. An increase in the delivery of free fatty acids reduces the ability of the insulin receptor substrates to activate phosphoinositide 3-kinase (PI 3-kinase) which results in the diminution of glucose transport and other events downstream of insulin receptor signaling [35]. HFD causes increased generation of 
Table 6: Activities of glucose-6-phosphatase, fructose-1, 6-bisphosphatase, and glucose-6-phosphate dehydrogenase in kidney tissues of control and experimental groups of rats

\begin{tabular}{llll}
\hline Groups & Glucose-6-phosphatase & Fructose-1, 6-bisphosphatase & Glucose-6-phosphate dehydrogenase \\
\hline Control & $362.10 \pm 19.00$ & $595.91 \pm 12.05$ & $612.10 \pm 28.97$ \\
Diabetic & $629.00 \pm 25.20^{\mathrm{a} *}$ & $881.26 \pm 32.82^{\mathrm{a} *}$ & $265.47 \pm 15.17^{\mathrm{a} *}$ \\
Diabetic+swertiamarin & $470.30 \pm 19.44^{\mathrm{b} *}$ & $697.48 \pm 27.39^{\mathrm{b} *}$ & $407.34 \pm 19.14^{\mathrm{b} *}$ \\
Diabetic+metformin & $451.50 \pm 19.50^{\mathrm{*} *}$ & $659.34 \pm 30.16^{\mathrm{b} *}$ & $415.42 \pm 29.75^{\mathrm{b*}}$ \\
\hline
\end{tabular}

Units are expressed as: $\mu$ moles of Pi liberated $/ \mathrm{h} / \mathrm{mg}$ of protein for glucose-6-phosphatase and fructose-1, 6-bisphosphatase and $\mu \mathrm{moles}$ of NADPH $/ \mathrm{min} / \mathrm{mg}$ of protein for glucose-6-phosphate dehydrogenase. Values are given as mean \pm SEM for groups of six rats in each. One-way ANOVA followed by post-hoc test LSD. Statistical significance was compared within the groups as follows: ${ }^{a}$ Control rats, bdiabetic control rats. Values are statistically significant at *p<0.05. SEM: Standard error mean, LSD: Least significant difference

Table 7: Level of glycogen content and activities of glycogen synthase and glycogen phosphorylase in liver tissues of control and experimental groups of rats

\begin{tabular}{llll}
\hline Groups & Glycogen & Glycogen synthase & Glycogen phosphorylase \\
\hline Control & $60.00 \pm 3.45$ & $816.00 \pm 29.42$ & $509.20 \pm 32.00$ \\
Diabetic & $24.50 \pm 1.43^{\mathrm{a} *}$ & $450.80 \pm 23.30^{\mathrm{a} *}$ & $782.72 \pm 31.70^{\mathrm{a} *}$ \\
Diabetic+swertiamarin & $42.60 \pm 4.00^{\mathrm{b} *}$ & $708.11 \pm 28.10^{\mathrm{b} *}$ & $700.50 \pm 27.00^{\mathrm{b} *}$ \\
Diabetic+metformin & $44.29 \pm 2.60^{\mathrm{b} *}$ & $680.08 \pm 28.30^{\mathrm{b} *}$ & $620.01 \pm 33.10^{\mathrm{b} *}$ \\
\hline
\end{tabular}

Units are expressed as: $\mathrm{mg}$ of glucose/g wet tissue for glycogen, $\mu$ moles of UDP formed/h/mg protein for glycogen synthase, and $\mu$ moles Pi liberated/h/mg protein for glycogen phosphorylase. Values are given as mean \pm SEM for groups of six rats in each. One-way ANOVA followed by post-hoc test LSD. Statistical significance

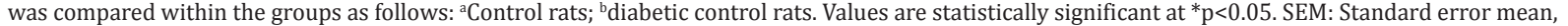
LSD: Least significant difference

reactive oxygen species, decreased mitochondrial function and closed calcium channel leading to decreased ATP synthesis, impaired glucose homeostasis, and ultimately $\beta$-cell dysfunction and death [36].

Diabetes mellitus is associated with loss of body weight, polyuria, polydipsia, and polyphagia. Impaired hepatic energy metabolism and a low energy state stimulate satiety center and increase food intake in T2DM [37]. Hepatic energy state has an influence on feeding behavior which in turn regulates body weight [38]. Initially, the rats fed with HFD for 2 weeks showed an increase in body weight due to the deposition of fat pads. Although the food intake of diabetic rats was increased during the entire experimental period, the weight gain was not statistically significant. The impaired glycemic control and unavailability of carbohydrate for energy production result in excessive catabolism of proteins to provide amino acids for gluconeogenesis, and this leads to muscle wasting and weight loss during insulin deficiency in diabetic rats [39]. Osmotic diuresis increases fluid intake in diabetic rats when compared to normal rats. However, oral administration of swertiamarin significantly normalized food and fluid intake suggesting an improvement in glycemic control in diabetic-treated rats.

OGTT measures the body's ability to utilize glucose, the body's main source of energy. An OGTT is a more sensitive measure of early abnormalities in glucose regulation than fasting plasma glucose [40]. OGTT is a primary test of immense value, in which the fasting plasma glucose concentration is estimated quantitatively to diagnose diabetes mellitus. Impaired glucose tolerance reflects increased hepatic gluconeogenesis and reduced uptake of glucose from the blood into skeletal muscle and adipose tissue following a meal [41]. The impaired glucose tolerance observed in the diabetic group of rats was altered to near normal by the treatment with swertiamarin which indicates the blood glucose lowering effect of Swertiamarin. Our results are in accordance with the findings of Patel et al. [42].

Blood glucose is a biochemical index for the diagnosis and prognosis of diabetes mellitus. In the present study, oral administration of swertiamarin decreased the levels of blood glucose and improved insulin sensitivity in HFD-STZ diabetic rats. The levels of plasma insulin were moderately decreased in experimental diabetic rats. Although the level was not decreased significantly, the insulin level in HFD-STZ diabetic rats could not stimulate glucose uptake into the cells due to the development of insulin-resistant state. There were an improved insulin sensitivity and a rise in insulin level in Swertiamarin-treated diabetic rats, suggesting that swertiamarin administration improved the insulin-sensitizing effect as well as improved glucose tolerance which may be probably due to improved pancreatic $\beta$-cell mass and function. The absence of sugar in the urine samples of swertiamarin-treated rats evidenced its renoprotective effect. The degree of hyperinsulinemia and insulin resistance was evidenced by fasting insulin levels and HOMA-IR. Diabetic rats exhibit severe insulin resistance and marked hyperglycemia. A significantly reduced HOMA-IR index in swertiamarintreated diabetic rats evidenced the efficacy of swertiamarin in restoring insulin sensitivity.

HbA1c is a reliable and reproducible biochemical parameter widely used to assess long-term maintenance of glycemic control. For longterm studies pertaining to diabetic complications, HbA1c is considered as an advantageous tool as it is not subjected to any momentary changes due to stress or some other acute factor. Thus, establishing the relationship between $\mathrm{HbA1c}$ and fasting glucose is considered as an important factor in the assessment of glucose homeostasis over the past 3 months [43].

HbA1c is formed as a post-synthetic modification of $\mathrm{HbA}$ throughout the life of the red blood cells. The amount of HbA1c formed reflects the status of carbohydrate metabolism of an individual. In uncontrolled or poorly controlled diabetes, there is an increased glycosylation of a number of proteins including hemoglobin [44]. HbA1c was found to increase in mice with diabetes mellitus to approximately $16 \%$, and the amount of increase is directly proportional to the fasting blood glucose level [45]. The levels of HbA1c strongly predict the risk of eye, kidney, and neural disease in diabetes mellitus and are regarded as a key target for the diagnosis and prognosis of diabetes-related complications [46]. During diabetes, the excess glucose present in the blood reacts with hemoglobin irreversibly. In the present study, rats treated with swertiamarin showed the significant reduction in the levels of HbA1c and an increased level of total hemoglobin in diabetic rats. This could be due to the beneficiary effect of swertiamarin in maintaining glucose homeostasis probably through the reduction of oxidative stress.

The liver plays an important role in the maintenance of normoglycemia by storing excessive glucose in the bloodstream and releases the same when the blood glucose level is physiologically low [47]. As a consequence of prolonged HFD feeding, the activity of enzymes involved 
in the glycolytic and pentose phosphate pathways is decreased while the activity of enzymes involved in gluconeogenic and glycogenolytic pathways is increased in the liver [48]. The reduction of HGP has been targeted as a strategy for diabetes treatment. The key enzymes that regulate carbohydrate metabolism are considered as a potential target for maintaining blood glucose levels. Due to STZ administration to HFD-fed rats, the pancreatic function is reduced to a greater extent and insulin is not secreted. Insulin regulates both uptake and utilization of glucose in target organs such as liver, kidney, skeletal muscle, and adipose tissue by controlling the activities of numerous metabolic enzymes.

Hexokinase is the key enzyme involved in the phosphorylation of glucose into glucose-6-phosphate [49]. Hexokinase insufficiency in diabetic mice could cause decreased glycolysis and decreased utilization of glucose for energy production. Oral administration of Swertiamarin to diabetic rats resulted in significant increase in the activity of hexokinase. The restored levels of plasma insulin and glucose in swertiamarin-treated HFD-STZ-induced diabetic rats may be attributed to the increased hepatic hexokinase activity.

PK is a ubiquitously expressed, rate-controlling, terminal glycolytic enzyme that catalyzes the conversion of phosphoenolpyruvate to pyruvate with the generation of ATP. The altered activity of PK during diabetic conditions could be expected to diminish the metabolism of glucose and ATP production. The decreased activity of PK readily accounts for the decreased rate of glycolysis and an increase in the gluconeogenic pathway which are considered as essential for the maintenance for normoglycemia.

LDH is a cytosolic enzyme which converts pyruvate to lactate during anaerobic glycolysis, which is subsequently converted to glucose through the gluconeogenic pathway. During diabetes mellitus, LDH activity was elevated due to the impairment of glucose-stimulated insulin secretion. Oral administration of swertiamarin, as well as metformin to HFD-STZ diabetic rats, showed a significant reduction in the LDH activity, probably due to the regulation of NAD+/NADH ratio by the oxidation of glucose.

Glucose-6-phosphate dehydrogenase catalyzes the rate-limiting step of the hexose monophosphate shunt and produces NADPH required for the maintenance of the levels of reduced glutathione, a non-enzymatic antioxidant [50]. The observed decrease in the activity of glucose6-phosphate dehydrogenase in the hepatic tissues of diabetic rats suggests a decrease in metabolism through the phosphogluconate oxidation pathway. In the present study, administration of swertiamarin significantly increased the activity of glucose-6-phosphate dehydrogenase in diabetic rats.

Glucose-6-phosphatase and fructose-1, 6-bisphosphatase are the regulatory enzymes in the gluconeogenic pathway. The pentose phosphate pathway (also called the phosphogluconate pathway and the hexose monophosphate shunt) is a process that generates NADPH and pentoses (5-carbon sugars). This pathway is considered as an alternative to the glycolytic process, and it primarily involves two phases, namely, oxidative and non-oxidative. In the oxidative phase, NADPH is generated and 5 -carbon sugars are synthesized in the non-oxidative phase. The decrease in the activity of fructose-1, 6-bisphosphatase has been linked to insulin resistance and obesity in experimental animal models of diabetes and suggests a principal role for fructose-1, 6-bisphosphatase in the gluconeogenic flux. In the present study, the reduced activities of both glucose-6-phosphatase and fructose-1, 6-bisphosphatase in hepatic tissues of diabetic rats were increased on oral administration of swertiamarin which revealed the reduced endogenous HGP.

Liver and skeletal muscle are the primary site of glucose disposal in the insulin-stimulated state. Glycogen is the storable form of glucose whose quantity in hepatic and skeletal muscle tissue is a reflection of insulin activity. The synthesis and degradation of glycogen are considered as reciprocally regulated pathways. Under physiological conditions, insulin enhances intracellular glycogen deposition by stimulating glycogen synthase and inhibiting glycogen phosphorylase activities. In the present study, the administration of swertiamarin to diabetic rats improved the glycogen content and normalized the altered activities of glycogen metabolizing enzymes in both the liver tissues, which is due to improved glucose utilization and storage. Oral administration of swertiamarin to carbohydrate-challenged mice significantly maintains the glycemic control through the regulation of carbohydrate metabolizing enzymes [51]

Insulin resistance due to HFD feeding may result in inadequate glucose uptake and ATP synthesis in the liver cells that make them fatigue. In the liver, defects in insulin-stimulated hepatic glycogen synthesis and increased rates of hepatic gluconeogenesis are the main factors that contribute to insulin resistance and fasting hyperglycemia [52]. However, oral treatment with swertiamarin regulates the key enzymes of carbohydrate metabolism, thereby maintaining glucose homeostasis.

\section{CONCLUSION}

Oral treatment with swertiamarin improves glucose homeostasis and possesses insulin-sensitizing effect in HFD-fed STZ-induced diabetic rats which is evident from the results of fasting blood glucose level, OGTT, plasma insulin, and HOMA IR values. The results of the present study also established the fact that swertiamarin increased the glycogen content by modulating the activities of glycogen metabolizing enzymes suggesting the effective utilization of glucose which in turn may be due to improved insulin sensitivity. Oral treatment with swertiamarin to experimental diabetic rats significantly improved the glycemic status by regulating the activities of key enzymes involved in the carbohydrate metabolism. Further studies are in progress to understand the molecular mechanisms involved in the action of swertiamarin in improving insulin sensitivity as well as its antidiabetic properties.

\section{SOURCE OF SUPPORT}

The research fellowship awarded by the University Grants Commission (UGC), Government of India, to the first author Mr. R. Selvam in the form of UGC-BSR is gratefully acknowledged.

\section{AUTHORS' CONTRIBUTIONS}

All the three authors have equal contribution in the manuscript.

\section{CONFLICTS OF INTEREST}

The authors declare no conflicts of interest.

\section{REFERENCES}

1. Arafat M, Salam A, Arafat O. The association of Type 2 diabetes with obesity and other factors: In multinational community. Int J Pharm Pharm Sci 2014;6:257-60

2. Edgerton DS, Kraft G, Smith M, Farmer B, Williams PE, Coate KC, et al. Insulin's direct hepatic effect explains the inhibition of glucose production caused by insulin secretion. JCI Insight 2017;2:e91863.

3. Prentki M, Nolan CJ. Islet $\beta$ cell failure in Type 2 diabetes. J Clin Invest 2006; $116: 1802-12$

4. Radziuk J, Pye S. Hepatic glucose uptake, gluconeogenesis and the regulation of glycogen synthesis. Diabetes Metab Res Rev 2001;17:250-72.

5. Nuttall FQ, Ngo A, Gannon MC. Regulation of hepatic glucose production and the role of gluconeogenesis in humans: Is the rate of gluconeogenesis constant? Diabetes Metab Res Rev 2008;24:438-58.

6. Kalsi A, Singh S, Taneja N, Kukal S, Mani S. Current treatments for Type 2 diabetes, their side effects and possible complementary treatments. Int J Pharm Pharm Sci 2015;7:13-8.

7. Gite VN, Pokharkar RD, Chopade VV, Takate SB. Hepatoprotective activity of Enicostemma axillare in paracetamol induced hepatotoxicity in albino rats. Int J Phar Life Sci 2010;1:50-3

8. Jaishree V, Badami S, Krishnamurthy PT. Antioxidant effect and hepatoprotective effect of ethyl acetate extract of Enicostemma axillare 
(Lam) Raynal against CCl4 induced liver injury in rats. Ind J Exp Bio 2010;48:896-904

9. Leelaprakash G, Dass S. In-vitro anti-inflammatory activity of methanol extract of Enicostemma axillare. Int J Drug Dev Res 2011;3:189-96.

10. Li HL, Peng XJ, He JC, Feng EF, Xu GL, Rao GX, et al. Development and validation of a LC-ESI-MS/MS method for the determination of swertiamarin in rat plasma and its application in pharmacokinetics. J Chromatogr B Analyt Technol Biomed Life Sci 2011;879:1653-8.

11. Vaidya H, Rajani M, Sudarsanam V, Padh H, Goyal R. Antihyperlipidaemic activity of swertiamarin, a secoiridoid glycoside in poloxamer-407-induced hyperlipidaemic rats. J Nat Med 2009;63:437-42.

12. Vaidya H, Prajapati A, Rajani M, Sudarsanam V, Padh H, Goyal RK, et al. Beneficial effects of swertiamarin on dyslipidaemia in streptozotocininduced Type 2 diabetic rats. Phytother Res 2012;26:1259-61.

13. Selvam R, Muruganantham K, Subramanian S. Phytochemical screening and evaluation of in vitro antioxidant efficacy of Enicostemma littorale Blume leaves extract. Int J Pharm Sci Rev Res 2018;49:113-20.

14. Srinivasan K, Viswanad B, Asrat L, Kaul CL, Ramarao P. Combination of high-fat diet-fed and low-dose streptozotocin-treated rat: A model for Type 2 diabetes and pharmacological screening. Pharmacol Res 2005;52:313-20.

15. Matthews DR, Hosker JP, Rudenski AS, Naylor BA, Treacher DF, Turner RC, et al. Homeostasis model assessment: Insulin resistance and beta-cell function from fasting plasma glucose and insulin concentrations in man. Diabetologia 1985;28:412-9.

16. Trinder P. Determination of blood glucose using an oxidase peroxidase system with a non-carcinogenic chromogen. J Clin Pathol 1969;22:158-61.

17. Drabkin DL, Austin JH. Spectrophotometric constants for common hemoglobin derivatives in human, dog and rabbit blood. J Biol Chem 1932;98:719-33

18. Nayak SS, Pattabiraman TN. Anew colorimetric method for the estimation of glycosylated hemoglobin. Clin Chim Acta 1981;109:267-74.

19. Brandstrup N, Kirk JE, Bruni C. The hexokinase and phosphoglucoisomerase activities of aortic and pulmonary artery tissue in individuals of various ages. J Gerontol 1957;12:166-71.

20. Pogson CI, Denton RM. Effect of alloxan diabetes, starvation and refeeding on glycolytic kinase activities in rat epididymal adipose tissue. Nature 1967;216:156-7.

21. King J. Colorimetric determination of serum lactate dehydrogenase. J Med Lab Tech 1959;16:265-9.

22. Koide H, Oda T. Pathological occurrence of glucose-6-phosphatase in serum in liver diseases. Clin Chim Acta 1959;4:554-61.

23. Gancedo JM, Gancedo C. Fructose-1, 6-bisphosphatase, phosphofructo kinase and glucose-6-phosphate dehydrogenase from fermenting and non-fermenting yeasts. Arch Mikrobiol 1971;76:132-8.

24. Ells HA, Kirkman HN. A colorimetric method for assay of erythrocytic glucose-6-phosphate dehydrogenase. Proc Soc Exp Biol Med 1961;106:607-9.

25. Leloir LF, Goldemberg SH. Glycogen synthetase from rat liver: $($ Glucose $) n+($ UDPG $) \rightarrow($ Glucose $) n+1+$ UDP. In: Colowick SP, Kalpan NO, editors. Methods in Enzymology. New York: Academic Press; 1962. p. $145-7$

26. Cornblath M, Randle PJ, Parmeggiani A, Morgan HE. Effects of phospholipids and anoxia on lactate production, glycogen content, and phosphorylase activity in the perfused isolated rat heart. J Biol Chem 1963;238:1592-97

27. Morales MA, Jabbagy AJ, Terenizi HR. Mutations affecting accumulation of Neurospora glycogen. News lett 1973;20:24-5.

28. Malireddy S, Kotha SR, Secor JD, Gurney TO, Abbott JL, Maulik G, et al. Phytochemical antioxidants modulate mammalian cellular Epigenome: Implications in health and disease. Antioxid Redox Signal 2012;17:327-39.

29. Ghibaudi L, Cook J, Farley C, Van Heek M, Hwa JJ. Fat intake affects adiposity, morbidity factors, and energy metabolism of SpragueDawley rats. Obes Res 2002;10:956-63.

30. Ikemoto S, Takahashi M, Tsunoda N, Maruyama K, Itakura H, Ezaki O, et al. High-fat diet-induced hyperglycemia and obesity in mice: Differential effects of dietary oils. Metabolism 1996;45:1539-46.

31. Wang H, Storlien LH, Huang XF. Effects of dietary fat types on body fatness, leptin, and ARC leptin receptor, NPY, and agRP mRNA expression. Am J Physiol Endocrinol Metab 2002;282:E1352-9.

32. Buettner R, Parhofer KG, Woenckhaus M, Wrede CE, KunzSchughart LA, Schölmerich J, et al. Defining high-fat-diet rat models: Metabolic and molecular effects of different fat types. J Mol Endocrinol 2006:36:485-501.

33. Mythili MD, Vyas R, Akila G, Gunasekaran S. Effect of streptozotocin on the ultrastructure of rat pancreatic islets. Microsc Res Tech 2004;63:274-81.

34. Surwit RS, Feinglos MN, Rodin J, Sutherland A, Petro AE, Opara EC, et al. Differential effects of fat and sucrose on the development of obesity and diabetes in C57BL/6J and A/J mice. Metabolism 1995;44:645-51.

35. Shulman GI. Cellular mechanisms of insulin resistance. J Clin Invest 2000;106:171-6.

36. Andrikopoulos S. Obesity and Type 2 diabetes: Slow down! Can metabolic deceleration protect the islet beta cell from excess nutrientinduced damage? Mol Cell Endocrinol 2010;316:140-6.

37. Cooper ME, Regnell SE. The hepatic cannabinoid 1 receptor as a modulator of hepatic energy state and food intake. Br J Clin Pharmacol 2014;77:21-30

38. Friedman MI. Obesity and the hepatic control of feeding behavior. Drug News Perspect 2007;20:573-8.

39. Panigrahi G, Panda C, Patra A. Extract of Sesbania grandiflora ameliorates hyperglycemia in high fat diet-streptozotocin induced experimental diabetes mellitus. Scientifica (Cairo) 2016;2016:4083568.

40. Singleton JR, Smith AG, Russell JW, Feldman EL. Microvascular complications of impaired glucose tolerance. Diabetes 2003;52:2867-73.

41. Robertson RP. Estimation of beta-cell mass by metabolic tests: Necessary, but how sufficient? Diabetes 2007:56:2420-4.

42. Patel TP, Soni S, Parikh P, Gosai J, Chruvattil R, Gupta S. Swertiamarin: An active lead from Enicostemma littorale regulates hepatic and adipose tissue gene expression by targeting PPAR- $\gamma$ and improves insulin sensitivity in experimental NIDDM rat model. Evid Based Complement Alternat Med 2013;358:673

43. Han BG, Hao CM, Tchekneva EE, Wang YY, Lee CA, Ebrahim B, et al. Markers of glycemic control in the mouse: Comparisons of 6-h- and overnight-fasted blood glucoses to HB Alc. Am J Physiol Endocrinol Metab 2008;295:E981-6.

44. Alberti KG, Press CM. The biochemistry and the complications of diabetes. In: Keen H, Jarrett J, editors. Complications of Diabetes. London: Edward Arnold; 1982. p. 231-e70.

45. Koenig RJ, Peterson CM, Jones RL, Saudek C, Lehrman M, Cerami A, et al. Correlation of glucose regulation and hemoglobin AIc in diabetes mellitus. N Engl J Med 1976;295:417-20.

46. Howlett J, Ashwell M. Glycemic response and health: Summary of a workshop. Am J Clin Nutr 2008;87:212S-216S

47. König M, Bulik S, Holzhütter HG. Quantifying the contribution of the liver to glucose homeostasis: A detailed kinetic model of human hepatic glucose metabolism. PLoS Comput Biol 2012;8:e1002577.

48. McAnuff MA, Omoruyi FO, Morrison EY, Asemota HN. Changes in some liver enzymes in streptozotocin-induced diabetic rats fed sapogenin extract from bitter yam (Dioscorea polygonoides) or commercial diosgenin. West Indian Med J 2005;54:97-101.

49. Laakso M, Malkki M, Deeb SS. Amino acid substitutions in hexokinase II among patients with NIDDM. Diabetes 1995;44:330-4.

50. Xu Y, Osborne BW, Stanton RC. Diabetes causes inhibition of glucose6-phosphate dehydrogenase via activation of PKA, which contributes to oxidative stress in rat kidney cortex. Am J Physiol Renal Physiol 2005;289:F1040-7.

51. Ahamad J, Hassan N, Amin S, Mir SR. Swertiamarin contributes to glucose homeostasis via inhibition of carbohydrate metabolizing enzymes. J Natl Remed 2016;16:125-30

52. Erion DM, Shulman GI. Diacylglycerol-mediated insulin resistance. Nat Med 2010;16:400-2. 